\title{
Limitation of pencil beam convolution (PBC) algorithm for photon dose calculations in inhomogeneous medium
}

\author{
Tesering Dorje \\ National Hospital, Gongphel Lam, Thimpu, Bhutan \\ Email address: \\ tesering_dorje@rediffmail.com
}

To cite this article:

Tesering Dorje. Limitation of Pencil Beam Convolution (PBC) Algorithm for Photon Dose Calculations in Inhomogeneous Medium. Journal of Cancer Treatment and Research. Vol. 2, No. 1, 2014, pp. 1-4. doi: 10.11648/j.jctr.20140201.11

\begin{abstract}
Purpose: The main purpose of this study is to investigate the accuracy of pencil beam convolution (PBC) algorithm when high-density inhomogeneity is involved along the photon beam path. This study will help the PBC users understand the limitation of PBC during the treatment planning of real cancer treatment plans, especially when tumor is located beyond high-density tissue such as bone. Methods: Inhomogeneous phantom $(30 \mathrm{~cm}$ x $30 \mathrm{~cm}, 17 \mathrm{~cm}$ deep $)$ with a 5 $\mathrm{cm}$ thick solid water as the top layer followed by $5 \mathrm{~cm}$ thick PVC and $7 \mathrm{~cm}$ solid water was manufactured for depth dose calculations and measurements. Data were obtained beyond PVC medium for three field sizes: $5 \times 5 \mathrm{~cm}^{2}, 10 \times 10 \mathrm{~cm}^{2}$, and $20 \times 20 \mathrm{~cm}^{2}$. Dose calculations were performed using PBC and measurements were done using chamber. Measured and calculated data were compared against each other. Results: PBC produced dose prediction errors beyond high density medium by $3.7 \%$ to $7.3 \%$ for field size $5 \times 5 \mathrm{~cm}^{2}$, by $4.8 \%$ to $6.9 \%$ for field size $10 \times 10 \mathrm{~cm}^{2}$, and by $5.9 \%$ to $7.3 \%$ for field size $20 \times 20 \mathrm{~cm}^{2}$. The results of this study, however, showed no clear dependency on the field size. Similarly, difference between the $\mathrm{PBC}$ and measurements did not show a clear trend when results at various points were compared with each other. Conclusion: PBC can overestimate the dose by up to $7.3 \%$ beyond high-density medium. High density materials such metallic immobilization devices must be avoided in the beam path during the patient treatment.
\end{abstract}

Keywords: Dose Calculation, PBC, Inhomogeneity Correction

\section{Introduction}

The primary goal of radiation therapy for cancer treatment is to deliver maximum dose to the target and reduce dose to the critical structures. In order to obtain better tumor control, an accuracy of dose delivery is very important in radiation therapy. One of the factors that can affect the dose delivery is the dose calculation algorithm used for dose computations in the treatment planning system (TPS). To meet the International Commission on Radiation Units (ICRU) criteria, dose calculation accuracy must be within 2-3\%. [1] Pencil beam convolution (PBC) algorithm is one of the commonly used algorithms for treatment planning in radiation therapy. PBC calculates dose distribution in infinitesimally narrow pencil beams and dose deposition kernels are derived from data obtained from the measurements in water. [2, 3] Furthermore, each pencil beam is corrected by a factor accounting for differences in beam attenuation [4], whereas the inhomogeneity correction is applied using equivalent tissue air ratio (ETAR) method. However, the inhomogeneity correction in lateral direction of the pencil beams is not accounted. [5] The purpose of this study is to investigate the accuracy of PBC when high-density inhomogeneity is involved along the photon beam path. This study will help the PBC users understand the limitation of PBC during the treatment planning for real cancer patients, especially when tumor is located beyond high-density tissue such as bone.

\section{Methods and Materials}

\subsection{Central Axis Depth Dose Calculation}

An inhomogeneous phantom (Figure 1) was manufactured for the depth dose calculation purpose. Dose computation was performed at an interval of $1 \mathrm{~cm}$ after high-density interface for three field sizes: 5 x $5 \mathrm{~cm}^{2}, 10 \mathrm{x}$ $10 \mathrm{~cm}^{2}$ and $20 \times 20 \mathrm{~cm}^{2}$. All central axis depth dose calculations were done for 200 monitor units (MUs) with a dose calculation grid size of $2.5 \mathrm{~mm}$. The source to surface distance (SSD) was $100 \mathrm{~cm}$ and beam energy was $6 \mathrm{MV}$. 


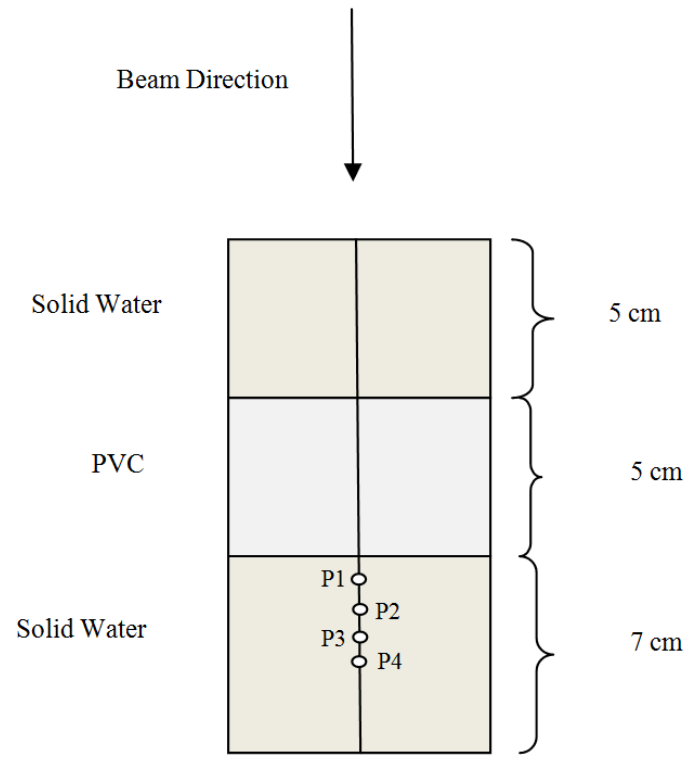

Figure 1: Schematic diagram of inhomogeneous phantom. Each point of interest (P1, P2, P3, and P4) is $1 \mathrm{~cm}$ apart, and P1 is $1 \mathrm{~cm}$ from PVC/Solid Water interface.

\subsection{Central axis Depth Dose Measurement}

For the depth dose measurements, 200 MUs were delivered using a linear accelerator (Model: $2100 \mathrm{CD}$ ) and electrometer readings at each depth of measurement were obtained using cylindrical ionization chamber. At a given depth, three readings were recorded, and the average reading was used for the analysis. Measurements were performed at $100 \mathrm{~cm}$ SSD for the identical beam parameters that were used for the PBC dose computations.

\subsection{Analysis}

Calculated and measured doses were converted to the percent depth doses (PDD) for analysis by normalizing all doses to the depth of maximum dose (dmax). At each point of interest (P1, P2, P3, and P4) as shown in Figure 1, difference in PDD between $\mathrm{PBC}$ and chamber was analyzed. Furthermore, the effect of field size on PDD was investigated too.

\section{Results and Discussion}

Table 1 shows the PDD calculated by the PBC and PDD measured by an ionization chamber for field sizes $5 \times 5 \mathrm{~cm}^{2}$, $10 \times 10 \mathrm{~cm}^{2}$, and $20 \times 20 \mathrm{~cm}^{2}$. For all points of interest $(\mathrm{P} 1$ - P4), it was observed that the PBC overestimated the PDD when compared to the measurements. For field size 5 × 5 $\mathrm{cm}^{2}$, PDD overestimation was by $3.7 \%$ to $7.3 \%$, whereas the for field size $10 \times 10 \mathrm{~cm}^{2}$, PDD overestimation was from $4.8 \%$ to $6.9 \%$. (Table 1 and Figure 2) Similarly, the PDD overestimation was found to be from $5.9 \%$ to $7.3 \%$ for field size $20 \times 20 \mathrm{~cm}^{2}$. (Table 1 and Figure 2)

Table 1: Comparison between the PBC percent depth dose (PDD) and measured/chamber PDD for field sizes $5 \times 5 \mathrm{~cm}^{2}, 10 \times 10 \mathrm{~cm}^{2}$, and $20 \times 20 \mathrm{~cm}^{2}$.

\begin{tabular}{|c|c|c|c|c|c|c|c|c|c|}
\hline \multicolumn{10}{|c|}{ PDD } \\
\hline & \multicolumn{3}{|c|}{$5 \times 5 \mathrm{~cm}^{2}$} & \multicolumn{3}{|c|}{$10 \times 10 \mathrm{~cm}^{2}$} & \multicolumn{3}{|c|}{$20 \times 20 \mathrm{~cm}^{2}$} \\
\hline & PBC & Chamber & $\mathrm{D}$ & PBC & Chamber & $\mathrm{D}$ & PBC & Chamber & $\mathrm{D}$ \\
\hline P1 & 53.6 & 51.7 & 3.7 & 58.4 & 55.2 & 5.8 & 62.7 & 58.9 & 6.5 \\
\hline P2 & 50.3 & 47.8 & 5.2 & 53.5 & 50.8 & 5.3 & 59.1 & 55.8 & 5.9 \\
\hline P3 & 47.1 & 44.4 & 6.1 & 50.8 & 47.5 & 6.9 & 55.9 & 52.1 & 7.3 \\
\hline P4 & 43.9 & 40.9 & 7.3 & 48.5 & 46.3 & 4.8 & 52.8 & 49.5 & 6.7 \\
\hline
\end{tabular}

$\mathrm{D}=$ Difference in PDD, where ${ }_{D}=\left(\frac{P B C-\text { Chamber }}{\text { Chamber }}\right) \times 100$

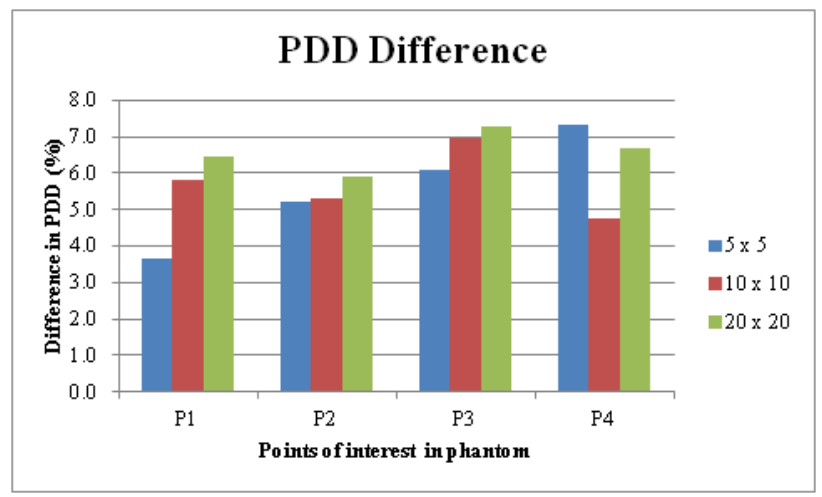

Figure 2: Difference in $P D D$ between the $P B C$ and measurements at $P 1$, $P 2, P 3$, and P4 for field sizes $5 \times 5 \mathrm{~cm}^{2}, 10 \times 10 \mathrm{~cm}^{2}$, and $20 \times 20 \mathrm{~cm}^{2}$ in inhomogeneous phantom (see Figure 1)
Several authors have investigated the accuracy of PBC in different experimental set up by comparing dose calculated by PBC with the measurements. For example, Gray et al [2] found that $\mathrm{PBC}$ could produce dose prediction errors greater than $2.5 \%$ when dose calculation is performed beyond large air gaps. Similarly, it was reported that difference in between PDD and measurements could be up to $6.7 \%$ beyond highdensity heterogeneity [6]. Those results [6] are consistent with the findings presented in this study. Several other authors have also reported dose prediction errors by dose calculation algorithms [7-12] when inhomogeneity correction is applied for photon dose calculations. Dose differences may also vary depending on the photon beam energy used for calculations. $[13,14,15]$ The results of this study, however, showed no clear dependency on the field size. Similarly, difference between the PBC and measurements did not show a clear trend when results at various points $(\mathrm{P} 1, \mathrm{P} 2, \mathrm{P} 3$, and $\mathrm{P} 4)$ were compared with each other. 
In PBC, final dose calculation is done based on the dose distribution in infinitesimally narrow pencil beams, and the dose deposition kernels, which are derived from the measurements done in the water medium, which is homogeneous. Since the real patient body may contain of different density, correction factor to each pencil beam needs to be applied, and this takes into account of the beam attenuation. However, dose from the adjacent pencil beams is ignored in each calculation. This could potential lead to dose prediction errors when the dose calculation needs to be done in the medium which may contain electron density that is significantly different than that of water. Hence, in the PBC, inhomogeneity correction is taken only in the beam direction. This could lead to unacceptable errors in patient dose calculation when treating a lung tumor or the chest wall, which has combination of high and low density heterogeneity.

It is well known fact that dose calculation algorithm is a major component in the treatment planning system (TPS) in radiation therapy. [8] Hence, it is imperative to have more accurate dose calculation engine for computations on the patient treatment plans. Otherwise, the treatment delivery will cause the under-dose of the tumor or overdose of the normal tissues. This will further cause unwanted treatment outcome in the patients.

The improvement in dose delivery technique using sophisticated techniques further complicates the accuracy of dose calculation algorithms. Some of the commonly used treatment techniques are intensity modulated radiation therapy (IMRT) and volumetric modulated arc therapy (VMAT). These techniques modulate the radiation beam to deliver conformal dose to the target. For example, during the dose delivery using VMAT technique, there is a simultaneous variation in the gantry speed, dose rate, and MLC leaf positions. Treatment techniques such as IMRT and $3 \mathrm{D}$ conformal radiation therapy (3DCRT) can also bring the variation in the dosimetric results. [16] Additionally, dose calculation accuracy can be also be dependent on the grid size used to calculate the cancer treatment plans.

In the recent years, there has been evolution of dose calculation algorithms such as analytical anisotropic algorithm (AAA), collapsed cone convolution (CCC) algorithm, and Acuros XB algorithm (AXB). [8, 15] The first two are model based algorithms, whereas the third one has been reported to be Monte Carlo based. It is known fact that Monte Carlo would be the perfect dose calculation algorithm, but due to higher calculation time, it is not feasible to use Monte Carlo algorithm clinically. Researchers have reported the dose errors from AAA and $\mathrm{CCC}$, especially in the high and low density media. Due to difference in the modeling approach within the dose calculation, it may not be possible to get the same calculated results among different algorithms. Among different commercially available dose calculation algorithms, the AXB appears to be more accurate one, and several researchers have reported superior accuracy of
AXB when compared to the AAA or PBC. $[8,15]$

Most of the treatment planning results are based on the physical dose-volume. There has been growing interest using the radiobiological models along with the dose calculation algorithms in the treatment planning system. [17] This will help the clinicians to relate the radiation dose to the radiobiological parameters such as tumor control probability and normal tissue complication probability.

This study was focused on the photon dose calculations. It is worth mentioning that proton dose calculations could prove to be superior to the photons. This is because of the physical properties of protons in terms dose deposition at the end of proton beam path with no exit dose and sharp lateral penumbra. $[18,19,20]$ Proton therapy could be more beneficial for the pediatric patients since there is less integral dose, and this can reduce the chance of secondary cancer. However, the researchers have reported the issue of neutron dose in proton therapy as well. [21-22]

In the experimental set up of this study, high-density material such as PVC will cause the beam attenuation, and photon beam is hardened as it passes through the $\mathrm{PVC}$ region. Dose difference between the $\mathrm{PBC}$ and the measurements seen in this study could be due to inaccurate estimation of beam attenuation and beam hardening as a result of high density medium. The accuracy of dose calculation algorithms is essential for tumor control; otherwise, inaccurate dose estimation may lead to tumor recurrence or higher normal tissue toxicities. The results presented in this study and other studies [2, 6-12] reveal that dose calculation algorithms have limitations in predicting accurate dose when photon beam passes through the high and low density heterogeneity. Hence, during the computed tomography (CT) simulation and patient treatment, it is essential to avoid the high and low density materials in the beam path prior to beam entering into the patient body. Further investigations on dose calculation algorithms are necessary, especially for different clinical scenarios.

\section{Conclusion}

PBC can overestimate the dose by up to $7.3 \%$ beyond high-density medium. High density materials such metallic immobilization devices must be avoided in the beam path during the patient treatment.

\section{References}

[1] International Commission on Radiation Units and Measurements (ICRU), "Determination of Absorbed Dose in a Patient Irradiated by Beams of X and Gamma Rays in Radiotherapy Procedures," ICRU Report, Bethesda, 1976.

[2] A. Gray, L. Oliver, P. Johnson, "The accuracy of the pencil beam convolution and anisotropic analytical algorithms in predicting the dose effects due to the attenuation from immobilization devices and large air gaps," Medical Physics, Vol. 36, No. 7, 2009; pp. 3181-91. DOI: http://dx.doi.org/10.1118/1.3147204 
[3] P. Storchi , L. Van Battum , E. Woudstra, "Calculation of a pencil beam kernel from measured photon beam data," Physics in Medicine and Biology, Vol. 44, No. 12, 1999; pp. 2917-28. DOI: http://dx.doi.org/10.1088/0031$9155 / 44 / 12 / 305$

[4] M. Sontag, J. Cunningham, "Corrections to absorbed dose calculations for tissue inhomogeneities," Medical Physics, Vol. 4, No. 5, 1977; pp. 431-6. DOI: http://dx.doi.org/10.1118/1.594329

[5] C. Hurkmans, T. Knoos, P. Nilsson, G. Svahn-Tapper, H. Danielsson, "Limitations of a Pencil Beam approach to photon dose calculations in the head and neck region," Radiotherapy Oncology, Vol. 37, No.1, 1995; pp. 74-80. DOI: http://dx.doi.org/10.1016/0167-8140(95)01609-K

[6] S. B. Rana, "Dose prediction accuracy of anisotropic analytical algorithm and pencil beam convolution algorithm beyond high density heterogeneity interface," South Asian Journal of Cancer, Vol. 2, No. 1, 2013; pp. 26-30. DOI: http://dx.doi.org/10.4103/2278-330X.105888

[7] T. P. Wong, P. E. Metcalfe, T. Kron, and T. G. Emeleus, "Radiotherapy x-ray dose distribution beyond air cavities," Australasian Physical \& Engineering Sciences in Medicine, Vol. 15, No. 3, 1992;pp. 138-146.

[8] L. Lu, "Dose calculation algorithms in external beam photon radiation therapy," International Journal of Cancer Therapy and Oncology, Vol. 1, No. 2, 2013; 01025. DOI: http://dx.doi.org/10.14319/ijcto.0102.5

[9] B. H. Shahine, M. S. A. L. Al-Ghazi, and E. El-Khatib, "Experimental evaluation of interface doses in the presence of air cavities compared with treatment planning algorithms," Medical Physics, Vol. 26, No. 3, 1999; pp. 350-355. http://dx.doi.org/10.1118/1.598526

[10] S. Oyewale, "Dose prediction accuracy of collapsed cone convolution superposition algorithm in a multi-layer inhomogenous phantom," International Journal of Cancer Therapy and Oncology, Vol. 1, No. 1, 2013; 01016. DOI: http://dx.doi.org/10.14319/ijcto.0101.6

[11] X. A. Li, C. Yu, and T. Holmes, "A systematic evaluation of air cavity dose perturbation in megavoltage x-ray beams," Medical Physics, Vol. 27, No. 5, 2000; pp. 1011-1017. http://dx.doi.org/10.1118/1.598966

[12] S. Rana, S. Pokharel, "Verification of dose calculation algorithms in a multi-layer heterogeneous phantom using films," Gulf Journal of Oncology, Vol. 1, No. 14, 2013; pp. 63-9.

[13] S. Hawke, A. Torrance, L. Tremethick, "Evaluation of planned dosimetry when beam energies are substituted for a fraction of the treatment course," International Journal of Cancer Therapy and Oncology, Vol. 1, No. 2, 2013; 01014. DOI: http://dx.doi.org/10.14319/ijcto.0102.4

[14] S. Pokharel, "Dosimetric impact of mixed-energy volumetric modulated arc therapy plans for high-risk prostate cancer.," International Journal of Cancer Therapy and Oncology, Vol. 1, No.1, 2013; 01011. DOI: http://dx.doi.org/10.14319/ijcto.0101.1

[15] S. Rana, "Clinical dosimetric impact of Acuros XB and analytical anisotropic algorithm (AAA) on real lung cancer treatment plans: review, " International Journal of Cancer Therapy and Oncology, Vol. 2, No, 1; 02019. DOI: http://dx.doi.org/10.14319/ijcto.0201.9

[16] S. Moorthy, H. Sakr, S. Hasan, et. al "Dosimetric study of SIB-IMRT versus SIB-3DCRT for breast cancer with breath-hold gated technique," International Journal of Cancer Therapy and Oncology, Vol. 1, No. 1, 2013, 010110. DOI: http://dx.doi.org/10.14319/ijcto.0101.10

[17] P. Mavroidis, "Clinical implementation of radiobiological measures in treatment planning. Why has it taken so long? "International Journal of Cancer Therapy and Oncology, Vol. 1, No. 1, 2013; 010109. DOI: http://dx.doi.org/10.14319/ijcto.0101.9

[18] W. Ulmer, "Notes of the editorial board on the role of medical physics in radiotherapy," International Journal of Cancer Therapy and Oncology, Vol. 1, No. 1, 2013; 01014. DOI: http://dx.doi.org/10.14319/ijcto.0101.4

[19] X. Zhang, Y. Li, X. Pan, et al. , "Intensity-modulated proton therapy reduces the dose to normal tissue compared with intensity-modulated radiation therapy or passive scattering proton therapy and enables individualized radical radiotherapy for extensive stage IIIB non-small-cell lung cancer: a virtual clinical study," Int J Radiat Oncol Biol Phys, Vol 77, No. 2, 2010;pp 357-66.

[20] S. Rana, H. Singh, "Impact of heterogeneities on lateral penumbra in uniform scanning proton therapy," International Journal of Cancer Therapy and Oncology, Vol. 1, No. 2, 2013; $01026 . \quad$ DOI: http://dx.doi.org/10.14319/ijcto.0102.6

[21] U. Schneider, S. Agosteo, E. Pedroni, J. Besserer, "Secondary neutron dose during proton therapy using spot scaninng," Int J Radiation Oncology Biol Phys, Vol 53, No. 1, 2002; pp 244-251.

[22] MR. Islam, "Secondary neutrons issue in proton radiotherapy-a brief report," International Journal of Cancer Therapy and Oncology, Vol. 2, No. 1, 2014; 02017.DOI: http://dx.doi.org/10.14319/ijcto.0201.7 